\title{
Effectiveness of focal muscle vibration on hemiplegic upper extremity spasticity in individuals with stroke: A systematic review
}

\author{
Anas R. Alashram ${ }^{\mathrm{a}, \mathrm{b}, *}$, Elvira Padua $^{\mathrm{c}}$, Cristian Romagnoli $^{\mathrm{d}}$ and Giuseppe Annino ${ }^{\mathrm{b}}$ \\ a School of Neuroscience, Faculty of Medicine and Surgery, University of Rome "Tor Vergata", Rome, Italy \\ ${ }^{\mathrm{b}}$ Department of Medicine Systems, University of Rome "Tor Vergata", Rome, Italy \\ ${ }^{\mathrm{c}}$ Department of Human Sciences and Promotion of the Quality of Life, San Raffaele Roma \\ Open University, Rome, Italy \\ ${ }^{\mathrm{d}}$ Science and Culture of Well-being and Lifestyle, Alma Mater University, Bologna, Italy
}

\begin{abstract}
.
BACKGROUND: The upper extremity spasticity following stroke limits individuals' activities of daily living. Focal muscle vibration (FMV) is a device producing vibration signals affecting the central nervous system.

OBJECTIVE: This systematic review was conducted to investigate the effects of FMV on individuals with stroke, and to identify the effective training protocol in reducing upper extremities spasticity post-stroke.

METHODS: We searched in SCOPUS, PEDro, PUBMED, REHABDATA, and Web of Science for randomized clinical trials (RCTs) and pseudo-RCTs published in English. The outcome measure included is the Modified Ashworth Scale (MAS). The methodological quality of the included trials was evaluated using the Cochrane Collaboration's instrument. Effect sizes were calculated.

RESULTS: Eight articles published from 2012 to 2019 were included in this systematic review. A total of 268 post-stroke patients, $28.73 \%$ of which were females, were included in all studies. The methodological quality for included studies ranged from moderate to high. FMV showed some evidence in reducing hemiplegic upper extremity spasticity in patients with stroke. CONCLUSIONS: The FMV may be an efficient intervention in reducing upper extremity spasticity in the stroke population. The efficient treatment protocol and dosage remain unclear. Additional randomized controlled trials are strongly needed to study the effects of FMV on spasticity in individuals with stroke.
\end{abstract}

Keywords: Vibration, upper extremity, stroke, spasticity, rehabilitation

\section{Introduction}

Stroke is a leading cause of morbidity and mortality worldwide and is frequently linked with upper

*Address for correspondence: Anas R. Alashram, Neuroscience PhD School, Department of Medicine Systems, Faculty of Medicine and Surgery, University of Rome "Tor Vergata", Montpellier street, 00133, Rome, Italy. E-mail: anasalashram@ gmail.com. ORCID: http://orcid.org/0000-0002-3066-3943. extremities persistent involvement (Broeks et al., 1999). According to recent research, the incidence of stroke worldwide ranged from 76 to 119 per 100,000 (Thrift et al., 2016). About $80 \%$ of stroke patients require upper extremity treatment (Friedman et al., 2014) with $40 \%$ of patients experience moderate to severe impairments in the upper extremity (Paquin et al., 2016.

More than $40 \%$ of stroke survivors experience this deficit chronically and more than $80 \%$ acutely 
(Cramer et al., 1997). Stroke individuals with mild to moderate upper extremity paresis have a good prognosis for functional recovery in the acute stage; only $71 \%$ of these patients perform at least some skills at 6 months following stroke (Nijland et al., 2010). Severely affected stroke patients have a poor prognosis with about $60 \%$ failing to achieve some activities of daily livings at 6 months post-stroke (Kwakkel et al., 2003, van Kuijk et al., 2008). Stroke is characterized by positive features include spasticity (O'Dwyer et al., 1996). About $39 \%$ of patients with first-ever stroke are spastic after 12 months (Watkins et al., 2002). Spasticity is defined as a velocity-dependent motor disorder characterized by an increase in tonic stretch reflexes (Lance, 1980).

The available treatment options for managing spasticity post stroke include numerous physical methods such as electrostimulation, splinting and muscle lengthening, use of spasmolytic drugs, surgeries such as muscle-tendon lengthening, neurectomy, and tenotomy, as well as various invasive procedures for neuromuscular blockade (Caldwell et al., 1969, Caldwell et al., 1974, Katz,1980, Glenn \& Elovic,1997, Keenan,1988, Skeil \& Barnes, 1994). Researches have shown that in order for rehabilitation intervention to be effective, treatment needs to be highly repetitive, raise afferent input and be functional, as well as engage the individual and encourage frequent practice (Pollock et al., 2014).

Focal muscle vibration (FMV) defined as a technique that applies a vibratory stimulus to a specific muscle or its tendon using a mechanical device. FMV generates the Ia inputs as a consequence of the activation primary ending of the muscle spindle (Roll et al., 1989), leading to alteration of corticospinal pathways (Styvers et al., 2003). Many studies reported an increase excitability in the primary motor cortex following low amplitude FMV when applied to the intrinsic hand muscles and flexor carpi radialis muscle in healthy subjects (Rosenkranz et al., 2003). Concerning individuals with stroke, several studies were published recently. One of these studies demonstrated a reduction in elbow joint spasticity after exposed to FMV (Annino et al., 2019). While another study by Celletti et al., (2017) did not show that reduction.

There is a significant increase in researches that investigated the influences of FMV on spasticity among people with stroke in the past decade. Besides, no systematic review has yet been published that provides clear assent about the effects of focal muscle vibration on upper extremity post-stroke. Thus, the aims of this systematic review were to investigate the effects of FMV on spasticity of the upper extremity in individuals with stroke, and if possible, to identify the effective treatment protocol.

\section{Methods}

\subsection{Search strategy}

A systematic literature search of multiple databases; SCOPUS, PEDro, PUBMED, REHABDATA, and Web of Science, was conducted for articles randomized clinical trials (RCTs) and pseudo-RCTs published in English. The keywords used for the electronic search were (focal muscle vibration OR local muscle vibration OR segmental muscle vibration OR localized muscle vibration OR vibration OR muscle vibration) AND (stroke OR cerebrovascular accident OR cerebral hemorrhage OR cerebral ischemia) AND (upper limb OR upper extremity OR arm) AND (Spasticity OR hypertonia OR tone OR spastic). MeSH terms were used in relevant databases: (STROKE [MeSH] OR stroke* OR cerebrovascular* OR cerebral*) AND (Vibration [MeSH]) AND (Upper Extremity [MeSH] OR upper limb OR arm) AND (Muscle Spasticity [MeSH]). No restriction in time was applied to the search strategy that was conducted in February 2019. A summary of the literature search strategy is presented in Fig. 1.

\subsection{Study selection}

We included the articles in this review if they were: published in the English language, used focal muscle vibration as a treatment intervention, classified as Randomized Clinical Trials (RCTs) or Pseudo-RCTs, assessed tone in the upper extremity, investigated individuals with the confirmed diagnosis for stroke. We excluded any articles which assessed individuals with other vibration modalities (i.e. whole body vibration) (Fig. 1).

\subsection{Methodological quality}

Two reviewers assessed the selected studies independently for the methodological quality using the Cochrane Collaboration's tool for evaluating the risk of bias (Higgins et al., 2011). The Cochrane tool has become the standard method to assess the risk of bias in randomized clinical trials (Jørgensen et al., 2016). The items include random sequence generation, allo- 


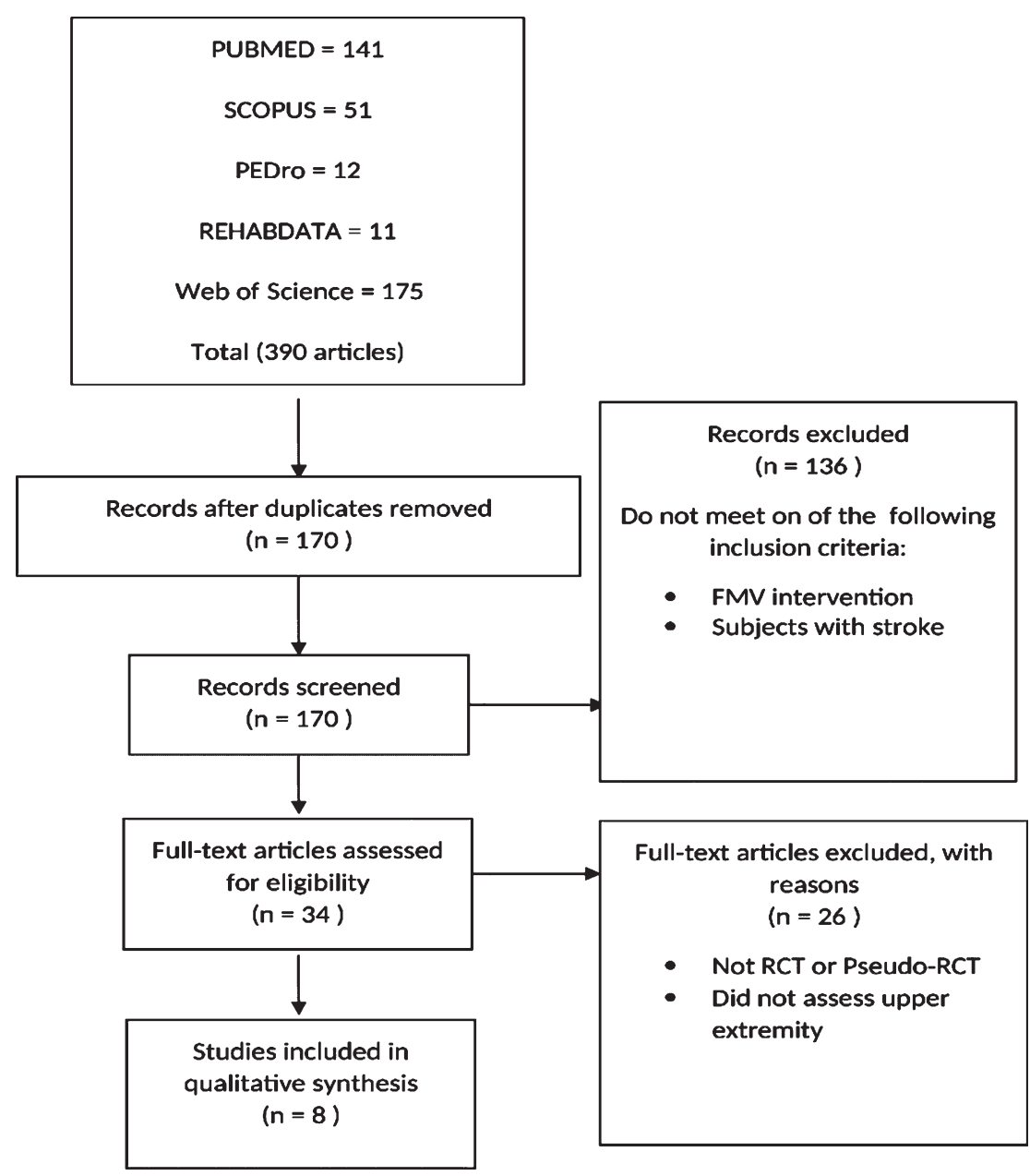

Fig. 1. Summary of literature review process.

cation concealment, blinding, incomplete outcome data, selective reporting, other bias (Higgins et al., 2008). Any disagreement was resolved through discussion with the third author. Table 1 displays the methodological quality assessment.

\subsection{Data extraction}

One reviewer has extracted the data, which was then checked by another. Any disagreements were resolved through discussion with the third author. Data extracted from the selected studies were: participant characteristics (number, gender, mean age, type of stroke, hemiplegic side, time since stroke), parameters (apparatus, frequency, amplitude, force, rest interval, target muscle, the status of muscle), experimental and control interventions (Table 2). The outcome measures are displayed in Table 3. Due to the heterogeneity treatment protocols and parameters and the inability to contact the authors of some studies, data were not pooled for meta-analysis. The effect size was estimated by dividing the variations between the mean of groups by the pooled of standard deviation (Ialongo, 2016). This study followed to all PRISMA guidelines (Liberati,2009).

\subsection{Outcome measure}

The modified Ashworth scale (MAS) designed to measure muscle tone and evaluate the level of spasticity. The MAS contains 6 items ranging from 0 to 4 , where 0 indicates have no increase in muscle tone. Item 1 indicates a slight increase in muscle tone at the end of the range of motion. Item 1+ indicates a slight increase in muscle tone throughout less than half of the range of motion. Item 2 indicates an increase in 
Table 1

Methodological quality assessment

\begin{tabular}{|c|c|c|c|c|c|c|}
\hline & $\begin{array}{c}\text { Random } \\
\text { sequence } \\
\text { generation } \\
\text { (selection bias) }\end{array}$ & $\begin{array}{c}\text { Allocation } \\
\text { concealment } \\
\text { (selection } \\
\text { bias) }\end{array}$ & $\begin{array}{c}\text { Blinding } \\
\text { (performance } \\
\text { and detection } \\
\text { bias) }\end{array}$ & $\begin{array}{l}\text { Incomplete } \\
\text { outcome data } \\
\text { (attrition } \\
\text { bias) }\end{array}$ & $\begin{array}{l}\text { Selective } \\
\text { reporting } \\
\text { (reporting } \\
\text { bias) }\end{array}$ & $\overline{\text { Other bias }}$ \\
\hline Caliandro et al., $2012^{30}$ & + & + & $?$ & + & + & + \\
\hline Celletti et al., $2017^{23}$ & + & + & + & + & $?$ & + \\
\hline Costantino et al., $2017^{34}$ & + & + & $?$ & + & + & + \\
\hline Paoloni et al., 2014 32 & + & $?$ & + & - & + & + \\
\hline Casale et al., $2014^{35}$ & + & $?$ & $?$ & - & + & + \\
\hline Noma et al., $2012^{33}$ & + & + & - & - & + & + \\
\hline Annino et al., $2019^{22}$ & + & - & - & + & + & + \\
\hline
\end{tabular}

Note: $+=$ low risk; ? = unclear risk; $-=$ high risk.

muscle tone through most of the range of motion. Item 3 indicates the difficult passive movement of the extremity. Item 4 indicates the extremity is rigid in flexion or extension (Bohannon \& Smith, 1987). Because the aim of the present review was to examine the influence of FMV on upper extremity spasticity, varied other measurements in included studies were not considered when interpreting findings.

\section{Results}

\subsection{Study selection}

The process of study selection for this review displayed in Fig. 1. A computerized search of PubMed (producing 141 articles), SCOPUS (51), PEDro (12), REHABDATA (11), and Web of Science (175) produced a total of 390 citations. After removing duplicates, 170 citations were reviewed. Out of those, 136 publications were excluded because their abstracts showed that they did not match the inclusion criteria.

Thirty-four publications were subjected to more detailed analysis because their abstracts did not reveal that they met the inclusion criteria. Twenty-six studies were excluded for the subsequent reasons: 1) used other forms of vibration, 2) did not assess upper extremity. A total of 8 studies were recognized for inclusion criteria in the present review.

\subsection{Patient characteristics}

The PICOS (Patients, Intervention, Control, Outcomes, and Subjects) approach was followed (Liberati, 2009). A total 268 post-stroke (Hemorrhagic $=36$, Ischemic $=232$ ), hemiplegic side $($ Left $=144$, Right $=118)$ were included. About
$28.73 \%$ of which were females were included in all studies, the mean age for all patients was 60 years old. Six studies included chronic stroke patients $(>12$ months) (Annino et al., 2019, Caliandro et al., 2012, Tavernese et al., 2013, Paoloni et al., 2014, Noma et al., 2012, Costantino et al., 2016), and one included acute stroke patients (Celletti et al., 2017), while one did not provide sufficient information about the stage of stroke (Casale et al., 2014). Table 2 shows patient characteristics for the included studies.

\subsection{Study design}

Three studies investigated FMV versus conventional physiotherapy (CPT) (Annino et al., 2019, Tavernese et al., 2013, Casale et al., 2014), two examined FMV versus placebo (Caliandro et al., 2012, Costantino et al., 2016), one investigated FMV versus CPT (Celletti et al., 2017), one examined FMV versus (placebo + CPT) (Paoloni et al., 2014), and one studied FMV versus stretching (control 1) and rest (control 2) (Noma et al., 2012).

The experimental interventions were FMV (Celletti et al., 2017, Caliandro et al., 2012, Noma et al., 2012, Costantino et al., 2016) or (FMV + CPT) (Annino et al., 2019, Tavernese et al., 2013, Paoloni et al., 2014, Casale et al., 2014). In terms of the treatment frequency, the frequency of treatment reneged from two (Celletti et al., 2017), to three (Annino et al., 2019, Caliandro et al., 2012, Costantino et al., 2016) or five (Tavernese et al., 2013, Paoloni et al., 2014, Casale et al., 2014) times per week. In terms of treatment protocol duration, the duration of the treatment protocol was one week (Caliandro et al., 2012), two weeks (Tavernese et al., 2013, Paoloni et al., 2014, Casale et al., 2014), four weeks (Costantino et al., 2016), six weeks (Celletti et al., 2017), or eight weeks 
Table 2

Participant characteristics and study design

\begin{tabular}{|c|c|c|c|}
\hline Study & Participant characteristics & Parameters & Intervention \\
\hline Caliandro et al., $2012^{30}$ & $\begin{array}{l}\text { Participants, } \mathrm{n}: 49 \\
\text { Gender M/F: } 34 / 15 \\
\text { Mean age: } 59.3 \\
\text { Stroke type, H/I, n: 33/17 } \\
\text { Hemiplegic side Rt/Lt, n : 26/23 } \\
\text { Time since stroke: }>12 \text { months }\end{array}$ & $\begin{array}{l}\text { Apparatus: CroSystem, Rome, } \\
\quad \text { Italy } \\
\text { Frequency }(\mathrm{Hz}): 100 \\
\text { Amplitude (mm): } 0.2-0.5 \\
\text { Force }(\mathrm{N}): 7-9 \\
\text { Rest interval: } 1 \mathrm{~min} \\
\text { Target muscle: Pectoralis minor, } \\
\text { Biceps brachii, } \\
\text { Flexor carpi muscle } \\
\text { Status of muscle: Contract }\end{array}$ & $\begin{array}{l}\text { Exp: FMV } 30 \mathrm{~min}(10 \mathrm{~min} / \mathrm{muscle}) \\
\quad \times 3 / \mathrm{wk} \times 1 \mathrm{wk} \\
\text { Con: Placebo }\end{array}$ \\
\hline Celletti et al., $2017^{23}$ & $\begin{array}{l}\text { Participants, } \mathrm{n}: 18 \\
\text { Gender M/F: } 12 / 6 \\
\text { Mean age: } 49.5 \\
\text { Stroke type, H/I, n: } 9 / 9 \\
\text { Hemiplegic side Rt/Lt, } \mathrm{n}: 9 / 9 \\
\text { Time since stroke: }<6 \text { months }\end{array}$ & $\begin{array}{l}\text { Apparatus: CroSystem, Rome, } \\
\quad \text { Italy } \\
\text { Frequency }(\mathrm{Hz}): 100 \\
\text { Amplitude (mm): } 0.2-0.5 \\
\text { Force }(\mathrm{N}): 7-9 \\
\text { Rest interval: } 1 \text { min } \\
\text { Target muscle: Pectoralis minor, } \\
\text { Biceps brachii, } \\
\text { Flexor carpi muscle } \\
\text { Status of muscle: Contract }\end{array}$ & $\begin{array}{l}\text { Exp: FMV } 30 \mathrm{~min}(10 \mathrm{~min} / \mathrm{muscle}) \\
\quad \times 2 / \mathrm{wk} \times 6 \mathrm{wk} \\
\text { Con: CPT } 30 \mathrm{~min} \times 2 / \mathrm{wk} \times 6 \mathrm{wk}\end{array}$ \\
\hline Costantino et al., $2017^{34}$ & $\begin{array}{l}\text { Participants, n: } 32 \\
\text { Gender M/F: } 21 / 11 \\
\text { Mean age: } 61.6 \\
\text { Stroke type, H/I, n: } 21 / 13 \\
\text { Hemiplegic side Rt/Lt, n: } 23 / 9 \\
\text { Time since stroke: }>12 \text { months }\end{array}$ & $\begin{array}{l}\text { Apparatus: VISSMAN, Rome, } \\
\text { Italy } \\
\text { Frequency }(\mathrm{Hz}): 300 \\
\text { Amplitude (mm): } 2 \\
\text { Force }(\mathrm{N}):- \\
\text { Rest interval: - } \\
\text { Target muscle: Triceps brachii, } \\
\text { Extensor carpi radialis longus } \\
\text { \& brevis } \\
\text { Status of muscle: Contract }\end{array}$ & $\begin{array}{l}\text { Exp: FMV } 30 \mathrm{~min}(10 \mathrm{~min} / \mathrm{muscle}) \\
\quad \times 3 / \mathrm{wk} \times 4 \mathrm{wk} \\
\text { Con: Placebo }\end{array}$ \\
\hline Tavernese et al., $2013^{31}$ & $\begin{array}{l}\text { Participants, n: } 44 \\
\text { Gender M/F: } 39 / 5 \\
\text { Mean age: } 58.6 \\
\text { Stroke type, H/I, n: } 44 / 0 \\
\text { Hemiplegic side Rt/Lt, n: 30/14 } \\
\text { Time since stroke: >12 months }\end{array}$ & $\begin{array}{l}\text { Apparatus: Horus, Akropolis, } \\
\text { Rome, Italy } \\
\text { Frequency (Hz): } 120 \\
\text { Amplitude (mm): } 10 \\
\text { Force (N): - } \\
\text { Rest interval: } 10 \mathrm{sec} \\
\text { Target muscle: Biceps brachii, } \\
\text { Flexor carpi ulnaris } \\
\text { Status of muscle: - }\end{array}$ & $\begin{array}{l}\text { Exp: FMV } 30 \mathrm{~min}+\mathrm{CPT} \\
60 \mathrm{~min} \times 5 / \mathrm{wk} \times 2 \mathrm{wk} \\
\text { Con: } \mathrm{CPT} 60 \mathrm{~min} \times 5 / \mathrm{wk} \times 2 \mathrm{wk}\end{array}$ \\
\hline Paoloni et al., $2014^{32}$ & $\begin{array}{l}\text { Participants, } \mathrm{n}: 22 \\
\text { Gender M/F: } 13 / 9 \\
\text { Mean age: } 60.5 \\
\text { Stroke type, H/I, n: } 22 / 0 \\
\text { Hemiplegic side Rt/Lt, n: } 14 / 8 \\
\text { Time since stroke:>12 months }\end{array}$ & $\begin{array}{l}\text { Apparatus: Horus, Akropolis, } \\
\quad \text { Rome, Italy } \\
\text { Frequency }(\mathrm{Hz}): 120 \\
\text { Amplitude }(\mathrm{mm}): 10 \\
\text { Force }(\mathrm{N}) \text { - } \\
\text { Rest interval: } 10 \mathrm{sec} \\
\text { Target muscle: Biceps brachii, } \\
\text { Flexor carpi ulnaris } \\
\text { Status of muscle: - }\end{array}$ & $\begin{array}{l}\text { Exp: FMV } 30 \mathrm{~min}+\mathrm{CPT} \\
60 \mathrm{~min} \times 5 / \mathrm{wk} \times 2 \mathrm{wk} \\
\text { Con: Placebo }+ \text { CPT } 60 \mathrm{~min} \\
\times 5 / \mathrm{wk} \times 2 \mathrm{wk}\end{array}$ \\
\hline Casale et al., $2014^{35}$ & $\begin{array}{l}\text { Participants, n: } 30 \\
\text { Gender M/F: } 18 / 12 \\
\text { Mean age: } 64.7 \\
\text { Stroke type, H/I, n: 30/0 } \\
\text { Hemiplegic side Rt/Lt, n: 4/26 } \\
\text { Time since stroke: - }\end{array}$ & $\begin{array}{l}\text { Apparatus: VI-BRA,Circle } \\
\text { Frequency }(\mathrm{Hz}): 100 \\
\text { Amplitude }(\mathrm{mm}): 2 \\
\text { Force }(\mathrm{N}):- \\
\text { Rest interval: }- \\
\text { Target muscle: Triceps brachii } \\
\text { Status of muscle: - }\end{array}$ & $\begin{array}{l}\text { Exp: FMV } 30 \mathrm{~min}+\mathrm{CPT} \\
60 \mathrm{~min} \times 5 / \mathrm{wk} \times 2 \mathrm{wk} \\
\text { Con: } \mathrm{CPT} 60 \mathrm{~min} \times 5 / \mathrm{wk} \times 2 \mathrm{wk}\end{array}$ \\
\hline
\end{tabular}


Table 2

(Continued)

\begin{tabular}{|c|c|c|c|}
\hline Study & Participant characteristics & Parameters & Intervention \\
\hline Noma et al., $2012^{33}$ & $\begin{array}{l}\text { Participants, n: } 36 \\
\text { Gender M/F: } 25 / 11 \\
\text { Mean age: } 60 \\
\text { Stroke type, H/I, n: } 36 / 0 \\
\text { Hemiplegic side Rt/Lt, n: 20/16 } \\
\text { Time since stroke: }>12 \text { months }\end{array}$ & $\begin{array}{l}\text { Apparatus: Thrive Co., Ltd, } \\
\quad \text { Osaka, Japan } \\
\text { Frequency }(\mathrm{Hz}): 91 \\
\text { Amplitude (mm): } 1 \\
\text { Force }(\mathrm{N}):- \\
\text { Rest interval: - } \\
\text { Target muscle: Biceps brachii, } \\
\text { Wrist flexors } \\
\text { Status of muscle: Stretched }\end{array}$ & $\begin{array}{l}\text { Exp: FMV } 5 \text { min } \\
\text { Con1: stretching } \\
\text { Con2: rest }\end{array}$ \\
\hline Annino et al., $2019^{22}$ & $\begin{array}{l}\text { Participants, } \mathrm{n}: 37 \\
\text { Gender M/F: } 29 / 8 \\
\text { Mean age: } 68.6 \\
\text { Stroke type, H/I, n: } 37 / 0 \\
\text { Hemiplegic side Rt/Lt, n: } 18 / 19 \\
\text { Time since stroke: }>12 \text { months }\end{array}$ & $\begin{array}{l}\text { Apparatus: }- \\
\text { Frequency }(\mathrm{Hz}): 30 \\
\text { Amplitude }(\mathrm{mm}): 2 \\
\text { Force }(\mathrm{N}):- \\
\text { Rest interval: } 1 \text { min } \\
\text { Target muscle: Triceps brachii } \\
\text { Status of muscle: Stretched }\end{array}$ & $\begin{array}{l}\text { Exp: FMV } 5 \mathrm{~min}+\mathrm{CPT} \\
30 \mathrm{~min} \times 3 / \mathrm{wk} \times 8 \mathrm{wk} \\
\text { Con: CPT } 30 \mathrm{~min} \times 3 / \mathrm{wk} \times 8 \mathrm{wk}\end{array}$ \\
\hline
\end{tabular}

FMV: focal muscle vibration, CPT: conventional physiotherapy, I/H: ischemic/hemorrhagic, Lt/Rt: Left/Right, Exp: experimental, Con: control, wk: week, min: minute, $\mathrm{n}$ : number, $\mathrm{M} / \mathrm{F}$ : male/female, sec: second.

(Annino et al., 2019). One study involved only one session (Noma et al., 2012). In terms of session duration, the session duration was 5 minutes (Noma et al., 2012), 30-35 minutes (Annino et al., 2019, Celletti et al., 2017, Tavernese et al., 2013, Costantino et al., 2016) or 90 minutes (Tavernese et al., 2013, Paoloni et al., 2014, Casale et al., 2014).

FMV apparatus varied between studies, CroSystem, Rome, Italy (Caliandro et al., 2012, Celletti et al., 2017), Horus, Akropolis, Rome, Italy (Tavernese et al., 2013, Paoloni et al., 2014), VISSMAN, Rome, Italy (Costantino et al., 2016), VIS-BRA,Circle (Casale et al., 2014), and Thriye CO., Ltd, Osaka, Japan (Noma et al., 2012). Concerning FMV total treatment duration, the FMV duration was 5 minutes (Annino et al., 2019, Noma et al., 2012) or 30 minutes (Celletti et al., 2017, Caliandro et al., 2012, Tavernese et al., 2013, Paoloni et al., 2014, Costantino et al., 2016), which ranged from 5 to 15 minutes for each treated muscle. In terms of FMV frequency, the frequency was set at $30 \mathrm{~Hz}$ (Annino et al., 2019), 91-100 Hz (Celletti et al., 2017, Caliandro et al., 2012, Noma et al., 2012, Casale et al., 2014), $120 \mathrm{~Hz}$ (Tavernese et al., 2013, Paoloni et al., 2014), and $300 \mathrm{~Hz}$ (Costantino et al., 2016). Regarding the amplitude, the FMV amplitude was set at 0.2-0.5 mm (Celletti et al., 2017, Caliandro et al., 2012), $1 \mathrm{~mm}$ (Noma et al., 2012), $2 \mathrm{~mm}$ (Annino et al., 2019, Costantino et al., 2016, Casale et al., 2014), or $10 \mathrm{~mm}$ (Tavernese et al., 2013, Paoloni et al., 2014). Concerning the force, the force was set at 7-9 N (Celletti et al., 2017, Caliandro et al., 2012). In terms of rest interval, it was 10 seconds (Tavernese et al., 2013, Paoloni et al., 2014), or one minute (Annino et al., 2019, Celletti et al., 2017, Caliandro et al., 2012).

Treated muscles were pectoralis minor (Celletti et al., 2017, Caliandro et al., 2012), biceps brachii (Celletti et al., 2017, Caliandro et al., 2012, Tavernese et al., 2013, Paoloni et al., 2014, Noma et al., 2012), triceps brachii (Annino et al., 2019, Costantino et al., 2016, Casale et al., 2014), extensor carpi radialis longus and brevis (Costantino et al., 2016), flexor carpi ulnaris (Tavernese et al., 2013, Paoloni et al., 2014), and wrist flexors (Noma et al., 2012). Regarding the status of muscles, it was contracted (Celletti et al., 2017, Caliandro et al., 2012, Costantino et al., 2016) or stretched (Annino et al., 2019, Noma et al., 2012). The summary of the study design and methodology for each study are listed in Table 2.

\subsection{Methodological quality}

Included studies ranged from moderate to high quality. All of the included studies have a low risk of bias in random sequence generation and other bias items. With the exception of Celletti et al., (2017), the studies have a low risk of bias in selective reporting (Table 1).

\subsection{Statistical analysis}

Statistical analysis between selected studies is different due to different study designs. One study used (ANOVA) for repeated measures to verify the differ- 
Table 3

Outcome measure

\begin{tabular}{|c|c|c|c|c|c|c|c|}
\hline Author & $\begin{array}{l}\text { Outcome } \\
\text { measure }\end{array}$ & Evaluated muscle/area & Assessment time & $\begin{array}{l}\text { Experimental group } \\
\text { Median }\end{array}$ & $\begin{array}{l}\text { Control group } \\
\text { Median }\end{array}$ & $\begin{array}{l}\text { Reported } \\
\text { effect }\end{array}$ & $\begin{array}{c}\text { Effect } \\
\text { size }\end{array}$ \\
\hline Caliandro et al., $2012^{30}$ & MAS & $\begin{array}{l}\text { Shoulder abduction and } \\
\text { aduction, flexion and } \\
\text { extension of elbow and } \\
\text { wrist }\end{array}$ & $\begin{array}{l}\text { Baseline, one week after } \\
\text { treatment, and one } \\
\text { month after treatment }\end{array}$ & $\begin{array}{l}\text { Pre-Shoulder } 1 \\
\text { Post-Shoulder } 0 \\
\text { Pre-Elbow } 2 \\
\text { Post-Elbow } 1 \\
\text { Pre-Wrist } 1 \\
\text { Post-Wrist } 1\end{array}$ & $\begin{array}{l}\text { Pre-Shoulder } 1 \\
\text { Post-Shoulder } 1 \\
\text { Pre-Elbow } 1 \\
\text { Post-Elbow } 1.5 \\
\text { Pre-Wrist } 2 \\
\text { Post-Wrist } 1.5\end{array}$ & $\begin{array}{l}= \\
= \\
=\end{array}$ & $\begin{array}{l}- \\
- \\
-\end{array}$ \\
\hline Celletti et al., $2017^{23}$ & MAS & $\begin{array}{l}\text { Flexion and extension of } \\
\text { elbow }\end{array}$ & $\begin{array}{l}\text { Baseline and } \\
\text { post-treatment }\end{array}$ & $\begin{array}{l}\text { Pre-Group (1) } 2 \\
\text { Post-Group (1) } 1.1\end{array}$ & $\begin{array}{l}\text { Pre-Group (2) } 2.35 \\
\text { Post-Group (2) } 1.6 \\
\end{array}$ & $=$ & - \\
\hline${ }^{*}$ Costantino et al., $2017^{34}$ & MAS & $\begin{array}{l}\text { Shoulder, elbow, and } \\
\text { wrist }\end{array}$ & $\begin{array}{l}\text { Baseline, post-treatment, } \\
\text { and after } 4 \text { weeks of } \\
\text { study protocol }\end{array}$ & $\begin{array}{l}\text { Pre-Shoulder } 1.59 \pm 1.33 \\
\text { Post-Shoulder } 1.12 \pm 1.05 \\
\text { Pre-Elbow } 2.00 \pm 1.22 \\
\text { Post-Elbow } 1.47 \pm 1.12 \\
\text { Pre-Wrist } 1.76 \pm 1.39 \\
\text { Post-Wrist } 1.18 \pm 1.24 \\
\end{array}$ & $\begin{array}{l}\text { Pre-Shoulder } 1.73 \pm 1.28 \\
\text { Post-Shoulder } 1.73 \pm 1.28 \\
\text { Pre-Elbow } 1.93 \pm 1.22 \\
\text { Post-Elbow } 1.87 \pm 1.19 \\
\text { Pre-Wrist } 1.67 \pm 1.35 \\
\text { Post-Wrist } 1.60 \pm 1.30 \\
\end{array}$ & $\begin{array}{l}+ \\
+ \\
+ \\
+\end{array}$ & $\begin{array}{l}0.11 \\
0.52 \\
0.10 \\
0.34 \\
0.10 \\
0.33 \\
\end{array}$ \\
\hline Tavernese et al., $2013^{31}$ & MAS & $\begin{array}{l}\text { Shoulder, elbow, and } \\
\text { wrist }\end{array}$ & $\begin{array}{l}\text { Baseline and } \\
\text { post-treatment }\end{array}$ & $\begin{array}{l}\text { Pre-Shoulder } 1.0 \\
\text { Pre-Elbow } 1.5 \\
\text { Pre-Wrist } 0.5\end{array}$ & $\begin{array}{l}\text { Pre-Shoulder } 2.0 \\
\text { Pre-Elbow } 2.0 \\
\text { Pre-Wrist } 1.0\end{array}$ & $\begin{array}{l}- \\
- \\
-\end{array}$ & $\begin{array}{l}- \\
- \\
-\end{array}$ \\
\hline Paoloni et al., $2014^{32}$ & MAS & $\begin{array}{l}\text { Shoulder, elbow, and } \\
\text { wrist }\end{array}$ & $\begin{array}{l}\text { Baseline and } \\
\text { post-treatment }\end{array}$ & $\begin{array}{l}\text { Pre-Shoulder } 1.0 \\
\text { Pre-Elbow } 1.0 \\
\text { Pre-Wrist } 1.0 \\
\end{array}$ & $\begin{array}{l}\text { Pre-Shoulder } 1.0 \\
\text { Pre-Elbow } 1.0 \\
\text { Pre-Wrist } 1.0 \\
\end{array}$ & $\begin{array}{l}+ \\
+ \\
+\end{array}$ & $\begin{array}{l}- \\
- \\
-\end{array}$ \\
\hline Casale et al., $2014^{35}$ & MAS & Biceps brachii & $\begin{array}{l}\text { Baseline, day number } 8 \text {, } \\
\text { and post-treatment }\end{array}$ & - & - & + & - \\
\hline Noma et al., $2012^{33}$ & MAS & $\begin{array}{l}\text { Biceps brachii } \\
\text { Flexors of wrist and } \\
\text { fingers }\end{array}$ & $\begin{array}{l}\text { Baseline, } 30 \text { minutes after } \\
\text { each intervention, and } \\
\text { post-treatment }\end{array}$ & $\begin{array}{l}\text { Pre-Elbow group (1) } 1.0 \\
\text { Pre-Wrist group (1) } 2.0\end{array}$ & $\begin{array}{l}\text { Pre-Elbow group (2) } 1.0 \\
\text { Pre-Wrist group (2) } 2.0 \\
\text { Pre-Elbow group (3) } 1.0 \\
\text { Pre-Wrist group (3) } 2.0\end{array}$ & $\begin{array}{l}+ \\
+ \\
\end{array}$ & $\begin{array}{l}- \\
- \\
\end{array}$ \\
\hline *Annino et al., $2019^{22}$ & MAS & Triceps & $\begin{array}{l}\text { Baseline and } \\
\text { post-treatment }\end{array}$ & $\begin{array}{l}\text { Pre-Elbow } 1.7 \pm 0.7 \\
\text { Post-Elbow } 1.1 \pm 0.8\end{array}$ & $\begin{array}{l}\text { Pre-Elbow } 1.5 \pm 0.9 \\
\text { Post-Elbow } 1.2 \pm 0.8\end{array}$ & $\begin{array}{l}+ \\
+\end{array}$ & $\begin{array}{l}0.25 \\
0.12\end{array}$ \\
\hline
\end{tabular}

MAS: Modified Ashworth Scale. ${ }^{*}$ : Presented as Mean \pm SD change, effect size was calculated. + , significant improvement in experimental group only. $=$, no significant differences between experimental and control group. 
ences of variables over time in both groups (Caliandro et al., 2012). Mann-Whitney U test was used to compare the central tendency measures (medians) of the MAS in both groups (Annino et al., 2019, Caliandro et al., 2012, Tavernese et al., 2013, Paoloni et al., 2014, Noma et al., 2012). Wilcoxon test was used to evaluate the change of MAS over time (Annino et al., 2019, Celletti et al., 2017, Tavernese et al., 2013, Paoloni et al., 2014, Noma et al., 2012). Moreover, one-way (ANOVA) was used to compare baseline demographic information and clinical characteristics of subjects between groups in addition to 2-way repeated measures (ANOVA) (Costantino et al., 2016). Shapiro-Wilk statistics test was used to assess the normality of the distribution of all variables (Casale et al., 2014). Paired $t$-test was used to compare the variables between assessment intervals (Casale et al., 2014).

\subsection{Outcome measures}

All of the included studies assessed the spasticity of the upper extremity in individuals with stroke using the Modified Ashworth Scale (MAS). Outcome measure details reported effect, and the effect size was presented in Table 3.

\subsection{Effect of FMV on upper extremity spasticity}

In the studies by Caliandro et al., (2012) and Celletti et al., (2017), the patients in both experimental and control groups demonstrated a reduction in the MAS scores (reduction in spasticity) of the upper extremity, however, with no significant difference between groups. The remaining studies demonstrated a significant reduction in MAS scores of the shoulder (Tavernese et al., 2013, Paoloni et al., 2014, Costantino et al., 2016), elbow (Annino et al., 2019, Tavernese et al., 2013, Paoloni et al., 2014, Noma et al., 2012, Costantino et al., 2016, Casale et al., 2014), and wrist (Tavernese et al., 2013, Paoloni et al., 2014, Noma et al., 2012, Costantino et al., 2016) in the experimental groups compared to the control groups.

\section{Discussion}

To our knowledge, this is the first systematic review aimed to examine the impacts of FMV in the treatment of upper extremities spasticity post stroke and to detect the effective training protocol. The initial findings showed that the FMV interventions may produce a reduction in hemiplegic upper extremity spasticity in patients with stroke. In agreement with our findings, Rosenkranz \& Rothwell, (2006) demonstrated to increase the primary motor cortex excitability following vibrated intrinsic hand muscles focally. FMV generates the Ia inputs as a consequence of the activation primary ending of muscle spindle (Roll et al., 1989). Alteration of the excitability of the corticospinal pathway result of activation of Ia inputs by FMV (Steyvers et al., 2003) by facilitating inputs and modulating of intracortical inhibiting to the primary motor cortex in the brain (Rosenkranz et al., 2003, Rosenkranz et al., 2006). The vibration of a specific muscle can increase the motor evoked potential (MEP) recorded from the muscle at rest (Mileva et al., 2009), suggesting the progress of corticospinal excitability changes during vibration (Rosenkranz et al., 2006, Smith \& Brouwer, 2005). Increased duration of cortical silent period (CSP) in flexor muscles of the forearm during vibration of the antagonist forearm extensors has been exhibited (Binder et al., 2009) and evidence strongly proposes that a period of pure sensory stimulation can affect motor cortical excitability (Ford et al., 1995).

Selected studies were superior in the research pyramid being randomized control trials or pseudorandomized control trials. Most studies had poor results in the blinding of participants and therapists (Annino et al., 2019, Caliandro et al., 2012, Tavernese et al., 2013, Paoloni et al., 2014, Noma et al., 2012, Costantino et al., 2016, Casale et al., 2014) leading to potential bias. While it is difficult to blind participants or therapists to vibration intervention, it is possible to blind the examiner to the vibration intervention. This is important to reduce the bias correlated with measurement based on outcomes (Higgins \& Green, 2008). Five studies have poor results in incomplete outcome data (Annino et al., 2019, Tavernese et al., 2013, Paoloni et al., 2014, Noma et al., 2012, Casale et al., 2014), and four studies have poor results in allocation concealment (Annino et al., 2019, Tavernese et al., 2013, Paoloni et al., 2014, Casale et al., 2014).

Six out of eight studies reported a positive effect of FMV in inhibiting hemiplegic upper extremity spasticity in patients with stroke (Annino et al., 2019, Tavernese et al., 2013, Paoloni et al., 2014, Noma et al., 2012, Costantino et al., 2016, Casale et al., 2014). Three of these studies demonstrated a reduction in the spasticity of the upper extremity in the experimental group ( $30 \mathrm{~min} \mathrm{FMV}+60 \mathrm{~min}$ $\mathrm{CPT} \times 5 / \mathrm{wk} \times 2 \mathrm{wk}$ ) compared to the control group 
(Tavernese et al., 2013, Paoloni et al., 2014, Casale et al., 2014). These studies were of moderate methodological quality and the sample sizes were large so the clinical conclusion can be confirmed. Moreover, Costantino et al., (2017) demonstrated a reduction in the spasticity of the upper extremity in the experimental group $(30 \mathrm{~min} F M V \times 3 / \mathrm{wk} \times 6 \mathrm{wk})$ compared to the control group. This study was of high methodological quality and the sample size was large, the effect sizes were small to moderate for wrist and elbow (0.10-0.34), and small to medium for shoulder joint (0.11-0.52), so the clinical significance of reported effects can be established. Furthermore, in a study by Annino et al., (2019), the participants demonstrated a reduction in upper extremity spasticity following ( $5 \mathrm{~min} \mathrm{FMV}+30 \mathrm{~min}$ $\mathrm{CPT} \times 3 /$ wk $\times 8$ wk) compared to the control group. This study was of moderate methodological quality and the sample size was large, the effect sizes were small to moderate $(0.12-0.25)$ so the clinical meaning of reported effects can be confirmed. As well, a study by Noma et al., (2012), he reported improvement after a single session of FMV, despite it was of high quality and involved large sample size, however, one study using a single session is not enough to generalize their results.

On the other hand, two studies reported a reduction in upper extremity spasticity in both experimental and control groups (Celletti et al., 2017, Caliandro et al., 2012). Although one of these studies was of high methodological quality, it involved small sample size, and the time since stroke $<6$ months (months) (Celletti et al., 2017), so we propose that the reduction in upper extremity spasticity of both groups was due to spontaneous recovery. As the sample size was also small, we cannot generalize the results. Regarding another study by Caliandro et al., (2012), we propose that three sessions per week for one week are not enough to produce a significant reduction in upper extremity spasticity.

This review was limited by articles published in English. This can lead to bias as studies with significant results have more chance to get published than studies that fail to report significant results (Egger \& Smith, 1998). Effect size not reported for some outcome measures because of insufficient data (Tavernese et al., 2013, Paoloni et al., 2014). We did not conduct meta-analysis because of the heterogeneity treatment protocols and dosages as well as the inability to contact the authors of many articles.

Due to the heterogeneity of studies, the effects of FMV on stroke patients were not fully understood.
Besides, the effective treatment protocol and dosage remain unclear. Furthermore, the selected studies did not report details about the vibration apparatuses, which makes it difficult to homogenize the outcomes. Reporting characteristics of the vibration device in future studies would allow specifying the outcomes. Further high quality studies with large sample sizes are definitely warranted.

\section{Conclusion}

In conclusion, the FMV may reduce the spasticity of the upper extremity in individuals with stroke if it is applied to the antagonist muscle. In fact, it seems that vibrated antagonist muscle may reduce the spasticity of the agonist muscle. We propose that adding the FMV into conventional physiotherapy intervention may produce superior effects than FMV intervention alone. No confirmed conclusion can be established regarding efficient treatment protocols and dosages due to the heterogeneity of the treatment interventions. Keeping in mind that there was a reduction in spasticity with various frequency ranges, it seems that the vibration frequency is not a discriminative factor in FMV intervention. We suppose that the treatment protocol and dosage could depend on the affected muscles and degree of spasticity more than other factors considered in the analysed studies. In this context, further high quality methodological trials with large sample size are strongly warranted to understand effects of FMV on upper extremity spasticity in stroke patients.

\section{Conflict of interest}

None to report.

\section{Funding}

None to report.

\section{References}

Annino, G., Alashram, A., Alghwiri, A., Romagnoli, C., Messina, G., \& Tancredi, V. et al. (2019). Effect of segmental muscle vibration on upper extremity functional ability poststroke. Medicine, 98(7), e14444. 
Binder, C., Kaya, A., \& Liepert, J. (2009). Vibration prolongs the cortical silent period in an antagonistic muscle. Muscle \& Nerve, 39(6), 776-780.

Bohannon, R., \& Smith, M. (1987). Interrater Reliability of a Modified Ashworth Scale of Muscle Spasticity. Physical Therapy, 67(2), 206-207.

Broesks, J., Lankhorst, G., Rumping, K., \& Prevo, A. (1999). The long-term outcome of arm function after stroke: results of a follow-up study. Disability And Rehabilitation, 21(8), 357-364.

Caldwell, C., \& Braun, R. (1974). Spasticity in the Upper Extremity. Clinical Orthopaedics And Related Research, 104, 80-91.

Caldwell, C., Wilson, D., \& Braun, R. (1969). Evaluation and Treatment of the Upper Extremity in the Hemiplegic Stroke Patient. Clinical Orthopaedics And Related Research, 1(63), 69-93.

Caliandro, P., Celletti, C., Padua, L., Minciotti, I., Russo, G., \& Granata, G. et al. (2012). Focal Muscle Vibration in the Treatment of Upper Limb Spasticity: A Pilot Randomized Controlled Trial in Patients With Chronic Stroke. Archives Of Physical Medicine And Rehabilitation, 93(9), 1656-1661.

Casale, R., Damiani, C., Maestri, R., Fundarò, C., Chimento, P., \& Foti, C. (2019). Localized $100 \mathrm{~Hz}$ vibration improves function and reduces upper limb spasticity: a double-blind controlled study. Eur J Phys Rehabil Med, 50(5), 495-504.

Celletti, C., Sinibaldi, E., Pierelli, F., Monari, G., \& Camerota, F. (2017). Focal Muscle Vibration and Progressive Modular Rebalancing with neurokinetic facilitations in post- stroke recovery of upper limb. Clin Ter, 168(1), 33-36.

Costantino, C., Galuppo, L., \& Romiti, D. (2016). Short-term effect of local muscle vibration treatment versus sham therapy on upper limb in chronic post-stroke patients: a randomized controlled trial. Eur J Phys Rehabil Med, 53(1), 32-40.

Cramer, S., Nelles, G., Benson, R., Kaplan, J., Parker, R., \& Kwong, K. et al. (1997). A Functional MRI Study of Subjects Recovered From Hemiparetic Stroke. Stroke, 28(12), 25182527.

Egger, M., \& Smith, G. (1998). meta-analysis bias in location and selection of studies. BMJ, 316(7124), 61-66.

Ford, B., Fahn, S., \& Pullman, S. (1995). Peripherally induced EMG silent periods. Normal physiology and disorders of motor control. Advances In Neurology, 67, 321-328.

Friedman, N., Chan, V., Reinkensmeyer, A., Beroukhim, A., Zambrano, G., Bachman, M., \& Reinkensmeyer, D. (2014). Retraining and assessing hand movement after stroke using the MusicGlove: comparison with conventional hand therapy and isometric grip training. Journal Of Neuroengineering And Rehabilitation, 11(1), 76.

Glenn, M., \& Elovic, E. (1997). Chemical Denervation for the Treatment of Hypertonia and Related Motor Disorders: Phenol and Botulinum Toxin. Journal Of Head Trauma Rehabilitation, 12(6), 40-62.

Higgins, J., \& Altman, D. Assessing Risk of Bias in Included Studies. Cochrane Handbook For Systematic Reviews Of Interventions, 187-241.

Higgins, J., Altman, D., Gotzsche, P., Juni, P., Moher, D., \& Oxman, A. et al. (2011). The Cochrane Collaboration's tool for assessing risk of bias in randomised trials. BMJ, 343(oct18 2), d5928-d5928.

Higgins, J., \& Green, S. (2008). Cochrane Handbook for Systematic Reviews of Interventions Version 5.0.2. [S.1.]: The Cochrane Collaboration.
Ialongo, C. (2016). Understanding the effect size and its measures. Biochemia Medica, 26(2), 150-163.

Jørgensen, L., Paludan-Müller, A., Laursen, D., Savović, J., Boutron, I., \& Sterne, J. et al. (2016). Evaluation of the Cochrane tool for assessing risk of bias in randomized clinical trials: overview of published comments and analysis of user practice in Cochrane and non-Cochrane reviews. Systematic Reviews, 5(1), 80.

Katz, R. (1988). Management of spasticity. Am J Phys Med Rehabil, 67(3), 108-116.

Keenan, M. (1988). Management of the Spastic Upper Extremity in the Neurologically Impaired Adult. Clinical Orthopaedics And Related Research, \&NA;(233), 116-125.

Kwakkel, G., Kollen, B., van der Grond, J., \& Prevo, A. (2003) Probability of Regaining Dexterity in the Flaccid Upper Limb. Stroke, 34(9), 2181-2186.

Lance, J. (1980). The control of muscle tone, reflexes, and movement: Robert Wartenbeg Lecture. Neurology, 30(12), 1303-1313.

Liberati, A., Altman, D., Tetzlaff, J., Mulrow, C., Gotzsche, P., \& Ioannidis, J. et al. (2009). The PRISMA statement for reporting systematic reviews and meta-analyses of studies that evaluate healthcare interventions: explanation and elaboration. $B M J$, 339(jul21 1), b2700-b2700.

Mileva, K., Bowtell, J., \& Kossev, A. (2009). Effects of lowfrequency whole-body vibration on motor-evoked potentials in healthy men. Experimental Physiology, 94(1), 103-116.

Nijland, R., van Wegen, E., Harmeling-van der Wel, B., \& Kwakkel, G. (2010). Presence of Finger Extension and Shoulder Abduction Within 72 Hours After Stroke Predicts Functional Recovery. Stroke, 41(4), 745-750.

Noma, T., Matsumoto, S., Shimodozono, M., Etoh, S., \& Kawahira, K. (2012). Anti-spastic effects of the direct application of vibratory stimuli to the spastic muscles of hemiplegic limbs in post-stroke patients: A proof-of-principle study. Journal of Rehabilitation Medicine, 44(4), 325-330.

O’Dwyer, N., Ada, L., \& Neilson, P. (1996). Spasticity and muscle contracture following stroke. Brain, 119(5), 1737-1749.

Paoloni, M., Tavernese, E., Fini, M., Sale, P., Franceschini, M., Santilli, V., \& Mangone, M. (2014). Segmental muscle vibration modifies muscle activation during reaching in chronic stroke: A pilot study. Neurorehabilitation, 35(3), 405-414.

Paquin, K., Crawley, J., Harris, J., \& Horton, S. (2016). Survivors of chronic stroke - participant evaluations of commercial gaming for rehabilitation. Disability And Rehabilitation, 38(21), 21442152.

Pollock, A., Farmer, S., Brady, M., Langhorne, P., Mead, G., Mehrholz, J., \& van Wijck, F. (2014). Interventions for improving upper limb function after stroke. Cochrane Database of Systematic Reviews, 12(11), CD010820.

Roll, J., Vedel, J., \& Ribot, E. (1989). Alteration of proprioceptive messages induced by tendon vibration in man: a microneurographic study. Experimental Brain Research, 76(1).

Rosenkranz, K., Pesenti, A., Paulus, W., \& Tergau, F. (2003). Focal reduction of intracortical inhibition in the motor cortex by selective proprioceptive stimulation. Experimental Brain Research, 149(1), 9-16.

Rosenkranz, K., \& Rothwell, J. (2006). Differences between the effects of three plasticity inducing protocols on the organization of the human motor cortex. European Journal of Neuroscience, 23(3), 822-829. 
Skeil, D., \& Barnes, M. (1994). The local treatment of spasticity. Clinical Rehabilitation, 8(3), 240-246.

Smith, L., \& Brouwer, B. (2005). Effectiveness of muscle vibration in modulating corticospinal excitability. The Journal of Rehabilitation Research And Development, 42(6), 787-794.

Steyvers, M., Levin, O., Van Baelen, M., \& Swinnen, S. (2003). Corticospinal excitability changes following prolonged muscle tendon vibration. Neuroreport, 14(15), 2001-2004.

Tavernese, E., Paoloni, M., Mangone, M., Mandic, V., Sale, P., Franceschini, M., \& Santilli, V. (2013). Segmental muscle vibration improves reaching movement in patients with chronic stroke. A randomized controlled trial. Neurorehabilitation, $32(3), 591-599$.
Thrift, A., Thayabaranathan, T., Howard, G., Howard, V., Rothwell, P., \& Feigin, V. et al. (2016). Global stroke statistics. International Journal of Stroke, 12(1), 13-32.

van Kuijk, A., Pasman, J., Hendricks, H., Zwarts, M., \& Geurts, A. (2008). Predicting Hand Motor Recovery in Severe Stroke: The Role of Motor Evoked Potentials in Relation to Early Clinical Assessment. Neurorehabilitation And Neural Repair, 23(1), 45-51.

Watkins, C., Leathley, M., Gregson, J., Moore, A., Smith, T., \& Sharma, A. (2002). Prevalence of spasticity post stroke. Clinical Rehabilitation, 16(5), 515-522. 